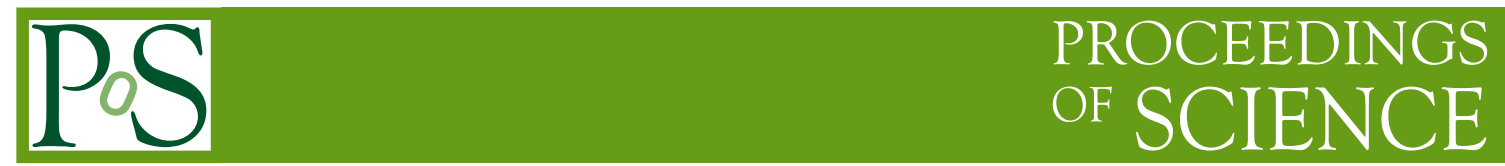

\title{
LCSR application to radiative tau decay
}

\author{
Anshika Bansal $^{a, b, *}$ and Namit Mahajan ${ }^{a}$ \\ a Physical Research Laboratory, \\ 380009, Ahmedabad, India \\ ${ }^{b}$ Indian Institute of Technology, \\ 382424, Gandhinagar, India \\ E-mail: anshika@prl.res.in, nmahajan@prl.res.in
}

The non-perturbative QCD effects involved in radiative tau decay $\left(\tau^{-} \rightarrow \pi^{-} v_{\tau} \gamma\right)$ are encoded in two form factors (FFs); vector $\left(F_{V}\right)$ and axial-vector $\left(F_{A}\right)$. These FFs are the same as those involved in radiative pion decay with the crucial difference that the square of the momentum transferred, $t$, between the $\pi-\gamma$ system is positive. This makes them timelike and can result in the production of real hadronic resonances. We calculate the analytical form for these FFs using the method of light-cone sum rules (LCSR) and present the decay width and the invariant mass spectrum of the $\pi-\gamma$ system. The structure-dependent parameter $\gamma$ (i.e., $\frac{F_{A}}{F_{V}}$ at zero momentum transfer) is found to be in good agreement with the experimental determination.

The Ninth Annual Conference on Large Hadron Collider Physics - LHCP2021

7-12 June 2021

Online

${ }^{*}$ Speaker 


\section{Introduction}

Being the heaviest lepton with mass $m_{\tau}=1776.86 \pm 0.12 \mathrm{MeV}$ [1], the tau has numerous decay channels, including hadronic channels. The hadronic decays of $\tau$ can be very helpful in understanding the hadronization of QCD currents. The branching ratio of the non-radiative decay $\left(\tau^{-} \rightarrow \pi^{-} v_{\tau}\right)$ is $10.82 \pm 0.05 \%$ [1]. A rough estimation suggests $\mathcal{B}\left(\tau^{-} \rightarrow \pi^{-} v_{\tau} \gamma\right) \approx \mathcal{B}\left(\tau^{-} \rightarrow\right.$ $\left.\rho v_{\tau}\right) \times \mathcal{B}\left(\rho^{-} \rightarrow \pi^{-} \gamma\right) \approx O\left(10^{-4}\right)-O\left(10^{-3}\right)$. Even though this branching ratio is not very small, the radiative decay has not been observed experimentally yet, which makes the study of this mode important. The amplitude of the radiative tau decay $\left(\tau \rightarrow \pi v_{\tau} \gamma\right)$ can be divided into two contributions: the internal bremsstrahlung (IB) and the structure dependent (SD) contributions. The former is due to the emission of a photon from the external particles ( $\tau$ and $\pi$ ) considering them to be pointlike (shown in (a) and (b) of Fig.(1)) while the latter captures the long-distance dynamics and can be parametrized by two time-like form factors (FFs); $F_{V}^{(\pi)}$ and $F_{A}^{(\pi)}$ (shown in (c) of Fig.(1)). A contact term (CT) (shown in (d) of Fig.(1)), which emerges as a consequence of gauge invariance, also needs to be included.

The ratio of these form factors when there is zero momentum transfer, known as the structure-

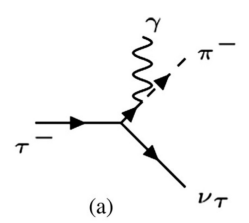

(a)

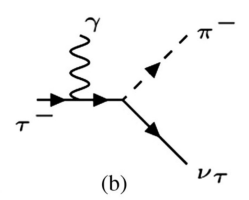

IB

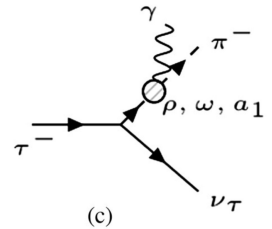

SD

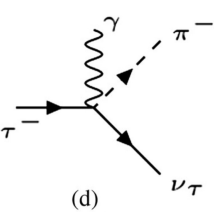

CT

Figure 1: Feynman diagrams showing different possibilities of photon emission.

dependent parameter (SDP) $\gamma$ (i.e., $\gamma=\frac{F_{A}^{(\pi)}(0)}{F_{V}^{(\pi)}(0)}$ ), provides information about the structure of the pion and hence becomes an important object of study. From the experimental determination of radiative pion decay, the SDP $\gamma$ is equal to $0.4685 \pm 0.0353$. In the literature, these FFs have been parametrised using the Breit-Wigner type resonances [2], the Light Front Quark Model [3] and the Resonance $\chi$ PT [4]. However, the use of vastly different approaches affected the predictions of the decay rate and spectrum and the value as well as the sign of $\gamma$. We calculate these FFs using the method of light-cone sum rules (LCSR) in a consistent way [5].

\section{Amplitude Calculation}

The amplitude of the radiative tau decay $\left(\tau^{-}\left(p_{1}\right) \rightarrow \pi^{-}\left(p_{2}\right) v_{\tau}\left(p_{3}\right) \gamma(k)\right)$ is given by

$$
\mathcal{A}\left(\tau^{-} \rightarrow \pi^{-} v_{\tau} \gamma\right)=\frac{G_{F}}{\sqrt{2}} V_{u d}\left\langle\pi^{-} v_{\tau} \gamma\left|\left(\bar{v}_{\tau} \Gamma^{\mu} \tau\right)\left(\bar{d} \Gamma_{\mu} u\right)\right| \tau^{-}\right\rangle
$$

where $\Gamma^{\mu}=\gamma^{\mu}\left(1-\gamma_{5}\right), G_{F}$ is the Fermi constant and $V_{u d}$ is the CKM element. The photon emission from $\tau$ can be trivially calculated using spinor QED. The photon emission from the pion is dictated by the hadronic matrix element

$$
T^{\alpha \mu}\left(p_{2}, k\right)=i \int d^{4} x e^{i k x}\left\langle\pi^{-}\left|T\left\{j_{e m}^{\alpha}(x) \bar{d} \Gamma^{\mu} u(0)\right\}\right| 0\right\rangle
$$


where $j_{e m}^{\alpha}(x)=Q_{\psi} \bar{\psi}(x) \gamma^{\alpha} \psi(x)=Q_{u} \bar{u} \gamma^{\alpha} u+Q_{d} \bar{d} \gamma^{\alpha}$. Using Lorentz and gauge symmetries it can be written in terms of two gauge-invariant scalar functions of $t=P^{2}=\left(p_{2}+k\right)^{2}$ as

$$
T^{\alpha \mu}\left(p_{2}, k\right)=F_{A}^{(\pi)}\left[g^{\alpha \mu}(P . k)-P^{\alpha} k^{\mu}\right]+i F_{V}^{(\pi)} \epsilon^{\alpha \mu \beta v} P_{\beta} k_{v}-i f_{\pi} g^{\alpha \mu}+i f_{\pi} \frac{P^{\alpha} P^{\mu}}{P . k} .
$$

Finally, the total amplitude reads as

$$
\mathcal{A}\left(\tau^{-} \rightarrow \pi^{-} v_{\tau} \gamma\right)=\mathcal{A}_{I B}+\mathcal{A}_{S D}
$$

$$
\begin{array}{r}
\mathcal{A}_{I B}=\frac{G_{F}}{\sqrt{2}} V_{u d}\left[i e f_{\pi} m_{\tau} \bar{u}_{v}\left\{\frac{\epsilon^{*} \cdot p_{1}}{p_{1} \cdot k}-\frac{\not k \xi^{*}}{2 p_{1} \cdot k}-\frac{\epsilon^{*} \cdot p_{2}}{p_{2} \cdot k}\right\}\left(1+\gamma_{5}\right) u_{\tau}\right], \\
\mathcal{A}_{S D}=\frac{G_{F}}{\sqrt{2}} V_{u d}\left[i e \epsilon^{* \alpha}\left(\bar{u}_{v} \Gamma^{\mu} u_{\tau}\right)\left(i F_{A}^{(\pi)}\left[g_{\alpha \mu}(P . k)-P_{\mu} k_{\alpha}\right]\right)-\left(F_{V}^{(\pi)} \epsilon_{\alpha \mu \beta \nu} P^{\beta} k^{v}\right)\right] .
\end{array}
$$

\section{Form factor calculation using LCSR}

The basic idea of QCD sum rules [6] is to derive the hadronic parameters using the analytical properties of the correlation function. In LCSR, instead of a vacuum to vacuum correlation function, one considers the correlation function between the vacuum and an on-shell state. The idea is to expand the product of the currents near the light-cone and express the bilocal operator in terms of the distribution amplitude as a series in twist (for more details, see [7-9]). At the leading twist (twist-2), the FFs turn out to be,

$$
F_{V}^{Q C D}(t)=\frac{i f_{\pi}}{3} \int_{0}^{1} d u \frac{\phi(u, \mu)}{t \bar{u}+k^{2} u}, \quad F_{A}^{Q C D}(t)=-i f_{\pi} \int_{0}^{1} d u \phi(u, \mu)\left(\frac{1-2 \bar{u}}{t \bar{u}+k^{2} u}\right)
$$

where, $\bar{u}=1-u$ and $\phi(u, \mu)$ is leading twist-2 distribution amplitude. Using the analytical properties of $T^{\alpha \mu}(P, k)$, one gets the following dispersion relation,

$$
\begin{aligned}
T^{\alpha \mu}\left(p_{2}, k\right) & =\frac{2 i m_{\rho} f_{\rho} \epsilon^{\alpha \lambda \beta v} g_{\lambda}^{\mu} p_{2 \beta} k_{v} F_{\rho \pi}\left(k^{2}\right)}{m_{\rho}^{2}-\left(p_{2}+k\right)^{2}-i m_{\rho} \Gamma_{\rho}}+\frac{i m_{a_{1}} f_{a_{1}}\left[2 p_{2} \cdot k g^{\alpha \mu}-2 p_{2}^{\alpha} k^{\mu}\right] G_{a_{1} \pi}\left(k^{2}\right)}{m_{a_{1}}^{2}-\left(p_{2}+k\right)^{2}-i m_{a_{1}} \Gamma_{a_{1}}} \\
& +\frac{1}{\pi} \int_{s_{0}^{h}}^{\infty} d s \frac{\operatorname{Im}\left\{T^{\alpha \mu}(s, k)\right\}}{s-k^{2}-i \epsilon} .
\end{aligned}
$$

Here, $s_{0}^{h}$ is the threshold of the lowest continuum state and $\Gamma_{\rho}\left(\Gamma_{a_{1}}\right), m_{\rho}\left(m_{a_{1}}\right)$ and $f_{\rho}\left(f_{a_{1}}\right)$ are the decay width, mass and decay constant of the $\rho\left(a_{1}\right)$ meson, respectively. $F_{\rho \pi}\left(G_{a_{1} \pi}\right)$ captures the physics of the transition of $\rho\left(a_{1}\right)$-meson to the $\pi$-meson. The contribution from the lowest resonance states i.e. $\rho, \omega$ and $a_{1}$-mesons is considered. The factor of 2 in the first term corresponds to the $\rho+\omega$ contribution. $F_{V}^{(\pi)}(t)\left(F_{A}^{(\pi)}(t)\right)$ gets contributions from the $\rho$ and $\omega\left(a_{1}\right)$ mesons. Using the duality approximation and Cauchy's integral, we find the sum rule for $F_{V}^{(\pi)}(t)$ and $F_{A}^{(\pi)}(t)$ to be

$$
\frac{2 m_{\rho} f_{\rho} F_{\rho \pi}\left(k^{2}\right)}{m_{\rho}^{2}-t}=\frac{i f_{\pi}}{3} \int_{0}^{u_{0}} d u \frac{\phi(u)}{t \bar{u}+k^{2} u}, \text { and } \quad \frac{2 i m_{a_{1}} f_{a_{1}} G_{a_{1} \pi}\left(k^{2}\right)}{m_{a_{1}}^{2}-t}=-i f_{\pi} \int_{0}^{u_{0}} d u \phi(u) \frac{1-2 \bar{u}}{t \bar{u}+k^{2} u} \text {, }
$$


respectively. Here, $u_{0}=\frac{s_{0}}{k^{2}+s_{0}}$. After applying Borel transformation on these sum rules, the final form of the FFs found to be

$$
\begin{aligned}
& F_{V}^{(\pi)}(t)=-i \frac{f_{\pi}}{3\left(m_{\rho}^{2}-t-i m_{\rho} \Gamma_{\rho}\right)} \int_{0}^{1} d u \frac{\phi(u)}{\bar{u}} e^{\frac{m_{\rho}^{2}}{M^{2}}}, \text { and } \\
& F_{A}^{(\pi)}(t)=-i \frac{f_{\pi}}{m_{a_{1}}^{2}-t-i m_{a_{1}} \Gamma_{a_{1}}} \int_{0}^{1} \frac{\phi(u)}{\bar{u}}(1-2 \bar{u}) e^{\frac{m_{a_{1}}^{2}}{M^{2}}} .
\end{aligned}
$$

Here, $\mathrm{M}$ is the Borel parameter. For the following computation, we have used the asymptotic and Chernyak-Zhitnisky form of the pion distribution amplitude [5].

\section{Results}

The IB contribution suffers from infrared (IR) divergences which can be taken care of by putting a threshold on the photon energy (50 MeV here). The structure dependent parameter, $\left.\gamma\right|_{C Z}(M=3.35 \mathrm{GeV})$ found to be 0.469 . The Borel parameter dependence of the form factors is shown in (a) of Fig.(2) and the invariant mass spectrum of the $\pi$ - system is shown in (b) of Fig.(2). The value of the normalised decay width $\bar{\Gamma}=\Gamma(\tau \rightarrow \pi v \gamma) / \Gamma(\tau \rightarrow \pi v))$ is found to be $(1.56 \pm 0.04) \times 10^{-2}$. The decay width is found to decrease with an increase in $\Gamma_{a_{1}}$. The uncertainties are found to be $\sim 10 \%$ and are dominated by SD contribution.

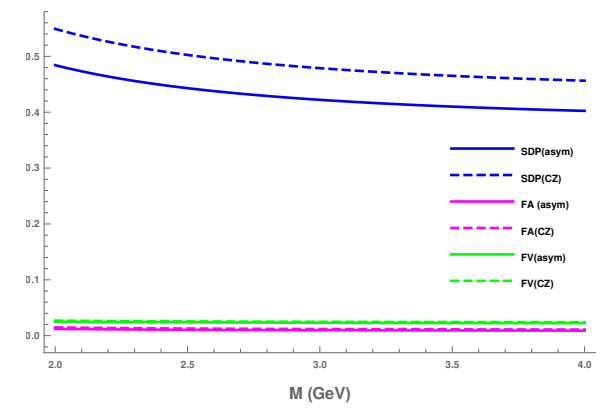

(a)

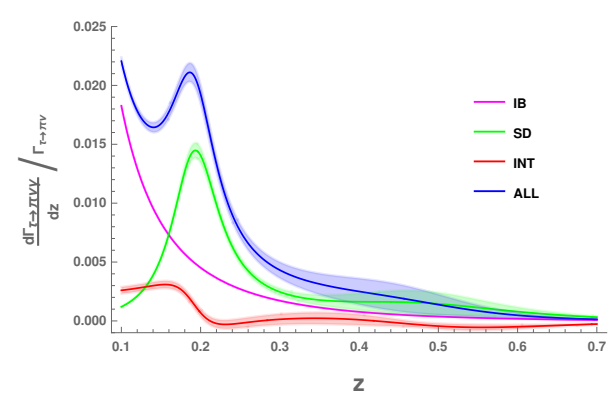

(b)

Figure 2: (a)The variation of $F_{V}^{(\pi)}(0)(\mathrm{Green}), F_{A}^{(\pi)}(0)$ (Magenta) and the structure dependent parameter (Blue) vs the Borel parameter $\mathrm{M}$ (in $\mathrm{GeV}$ ). (b) The invariant mass spectrum of the $\pi-\gamma$ system considering asymptotic distribution amplitude. $z$ is $\frac{t}{m_{\tau}^{2}}$. The shaded region shows the uncertainties.

\section{Conclusions and Discussions}

We have presented a detailed prediction for the decay rate and photon spectrum for radiative $\tau$ decay. A contact term appears as a consequence of gauge invariance. The decay includes two timelike FFs which are calculated in the framework of LCSR upto twist-2 accuracy. The structure dependent parameter was calculated and was found to be in good agreement with the experimental value (including the sign) obtained from the radiative pion decay. The normalised rate values were consistent with the study done using the resonance $\chi$ PT by Roig et. al [4]. This is the first application of LCSR to such a mode. A study of higher twist contribution and radiative corrections will be interesting. 


\section{References}

[1] M. Tanabashi et al. (Particle Data Group), 'Review of Particle Physics', Phys. Rev. D 98, 030001 (2018).

[2] R. Decker and M. Finkemeier, 'Radiative corrections to the decay $\tau \rightarrow \pi(K) v_{\tau}$ ', Phys. Lett. B 334, 199-202, (1994).

[3] C.Q. Geng and C.C. Lih, ' $\tau$ radiative decays in the light front quark model', Phys. Rev. D, 68, 093001 (2003).

[4] Zhi-Hui Guo and Pablo Roig, 'One meson radiative tau decays', Phys. Rev. D 82, 113016, (2010).

[5] Anshika Bansal and Namit Mahajan, 'Phenomenology of $\tau^{-} \rightarrow \pi^{-} v_{\tau} \gamma$ using light cone sum rules', Phys. Rev. D 103, 056017 (2021).

[6] Mikhail A. Shifman, A.I. Vainshtein, and Valentin I. Zakharov, 'QCD and Resonance Physics', Theoretical Foundations, Nucl. Phys. B 147, 385-447, (1979).

[7] Pietro Colangelo and A. Khodjamirian, 'QCD sum rules, a modern perspective', in At The Frontier of Particle Physics (World Scientific, 2000), pp. 1495-1576, https://doi.org/10.1142/9789812810458_0033, [arXiv: hep-ph/0010175].

[8] Vladimir M. Braun, 'Light cone sum rules', In $4^{\text {th }}$ International Workshop on Progress in Heavy Quark Physics, pages 105-118, 9 (1997).

[9] Mikhail A. Shifman, 'Snapshots of hadrons or the story of how the vacuum medium determines the properties of the classical mesons which are produced, live and die in the QCD vacuum', Prog. Theor. Phys. Suppl. 131, 1-71 (1998). 